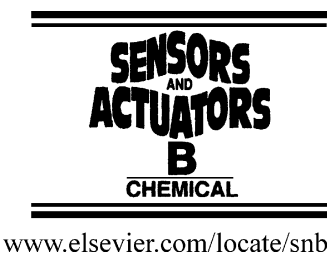

\title{
A surface acoustic wave sensor modified from a wireless transmitter for the monitoring of the growth of bacteria
}

\author{
Ku-Shang Chang a,b,*, Chen-Kai Chang ${ }^{\text {a,c }}$, Chien-Yuan Chen ${ }^{\mathrm{a}, * *}$ \\ a Department of Biochemical Science and Technology \& Graduate Institute of Microbiology and Biochemistry, National Taiwan University, Taipei, Taiwan \\ ${ }^{\mathrm{b}}$ Department of Food Science \& Graduate Institute of Biotechnology, Yuanpei University of Science and Technology, Hsinchu, Taiwan \\ ${ }^{\mathrm{c}}$ Department of Food Science and Technology, Chia Nan University of Pharmacy and Science, Tainan, Taiwan
}

Received 15 June 2006; received in revised form 26 January 2007; accepted 6 February 2007

Available online 11 February 2007

\begin{abstract}
This work presents a novel surface acoustic wave (SAW) sensor for the measurement of the microbial count in a biological culture. The signal translation interface of the sensor was modified using a common commercial wireless SAW transmitter design. A pair of conductive electrodes was inserted in a $314.5 \mathrm{MHz}$ SAW stabilized oscillator of the transmitter. Measurements were made by placing the electrodes within the culture solution of interest and measured the time required to identify readily detectable frequency changes (detection time, DT). The change of frequency was caused by the impedance change of the microbial metabolism. The calibration curve of detection times against density of Escherichia coli shows a linear correlation coefficient $\left(R^{2}=0.924\right)$ over the range of $10^{2}$ to $10^{7}$ cells $/ \mathrm{mL}$. A sample that contains $10^{2}$ cells $/ \mathrm{mL}$ of $E$. coli required a detection times (DT) of $7 \mathrm{~h}$, shorter than was required using instruments based on conventional conductance methods. The proposed ultra high frequency SAW sensor (314.5 MHz) shows a large total frequency change and gives a sharp inflexion at the DT but possesses the same stability compared with that of low frequency serial piezoelectric quartz crystal (PQC) sensor for the measurement of bacteria concentration. This sensor platform enabled real time monitoring of bacterial growth within a sealed opaque container. This sensor is potentially applicable to a remote query wireless measurement in hazardous environments when a suitable antenna device is adapted.
\end{abstract}

(C) 2007 Elsevier B.V. All rights reserved.

Keywords: Surface acoustic wave; SAW; Escherichia coli; Bacterial growth

\section{Introduction}

Conventional microbiological methods for determining bacteria counts are time-consuming, taking up to 2 days. Additionally, a large number of tests lead to great expense due to labor, material costs, and huge storage costs in the food industry. As a result, various rapid methods have been developed for the determination of bacteria counts in the past several decades, including ATP estimations [1], direct epifluorescent filter technique [2], amperometric biosensing method [3], immuno-biosensor assays [4], and electrical methods [5,6]. This work presents an ultra-

\footnotetext{
* Corresponding author. Tel.: +886 $35381183 x 8483$; fax: +886 35385353 .

** Corresponding author at: Department of Biochemical Science and Technology \& Institute of Microbiology and Biochemistry, National Taiwan University, No. 1, Sec. 4, Roosevelt Road, Taipei 106, Taiwan. Tel.: +8862 23630231x3256; fax: +886223661696 .

E-mail addresses: tommy.first@msa.hinet.net (K.-S. Chang), chenyuan@ntu.edu.tw (C.-Y. Chen).
}

high frequency surface acoustic wave (SAW; $314.5 \mathrm{MHz}$ ) sensor for the measurement of the microbial count in a biological culture. This type of acoustic biosensor could potentially offer a simple, low-cost and robust (repeatable) way to assay bacterial loads in complex liquids [7]. The availability of an ultra-high frequency also enables the design of wireless remote control functions. The ATP method and epifluorescent filter method are fast but complicated for handling. Strong electrochemical interference from oxidisable species in the media exposes a serious problem for the practical operation of amperometric biosensors. The immuno-biosensor assays encounter non-specific adsorption of protein during measurement. Electrical methods are simple but require expensive instruments. Impedance microbiology [8] was one of the earliest electrical methods for the detection of bacteria in foods, and has been developed as a rapid method that can detect bacteria within $24 \mathrm{~h}[9,10]$. It is based on the measurement of changes in electrical impedance of a medium or a reaction solution resulting from the growth of bacteria. The growth of microbial populations in culture changes the electrical 
properties of the culture medium. Substrates in microbiological growth media are generally uncharged or weakly charged. They are normally transformed into highly charged end products following the organism's normal metabolic pathways. Thus the electrical properties of the test medium will change during the growth of bacteria $[9,11]$. Simple examples include the conversion of glucose from a non-ionized substrate to two molecules of lactic acid with a corresponding increase in conductivity. Typically, changes in the electrical properties of the culture medium resulting from bacterial growth become detectable when the microbial density reaches $10^{6}$ to $10^{7}$ cells/mL $[12,13]$. The time required to identify readily detectable changes (detection time, DT) provides an approximate measure of the number of microbial organisms in the initial inoculums [14].

Two types of measurement techniques have proved useful in commercial applications: conductivity and impedance $[5,15,16]$. Devices based on these techniques monitor microbial metabolism in growth medium by immersing electrodes directly into the medium and measuring the permittivity and/or conductivity [17]. The impedance method was approved as an official method for the detection of Salmonella in foods by the Association of Analytical Communities (AOAC) [6]. Despite their widespread application, these techniques have many disadvantages including polarization of the probe electrodes, decreased sensitivity of the device in more conductive media and the high cost of the instruments [18]. Alternatively, surface acoustic wave (SAW) resonator impedance systems appear very attractive because they work at very high frequency [19], and thus electrode polarization is eliminated. Surface acoustic wave (SAW) resonator transmitters have been widely used for unlicensed applications for more than a decade. Applications include: automotive keyless entry, door and gate openers, wireless alarm sensors, bar code reader, and many others in wireless remote control, security and transmission areas. The SAW resonator stabilized oscillator provides a very stable fundamental mode frequency source at ultra-high frequency.

Yoa et al. developed a system consisting of a SAW resonator $(61 \mathrm{MHz})$ and a pair of conductive electrodes in the feedback circuit with a radio frequency amplifier [20,21]. Designs using SAW impedance systems have been applied to various bioanalytical fields concerning the (i) total salt concentration in serum [22], (ii) determination of urea [23], and (iii) pancreatic lipase [24]. The system can respond to changes in the capacitance and the conductance of the solution between the two electrodes, but its use to monitor bacterial growth has not been reported. Furthermore, there have been no studies of SAW impedance system using an ultra-high frequency resonator signal, which could improve sensitivity [25]. The availability of an ultra-high frequency also enables the design of wireless remote control functions [26].

This article describes the development of a sensor with a signal translation circuit, modified using a common commercial wireless SAW transmitter. The device consists of a pair of conductive electrodes inserted into a $314.5 \mathrm{MHz}$ SAW stabilized oscillator. The oscillator is characterized by its low cost, small size and energy-efficiency. We describe the application of this sensor for measurement of the number of microbes in a biologi- cal culture. The stability and sensitivity of proposed SAW sensor was also compared with that of low frequency PQC $(8.0 \mathrm{MHz})$ sensor [27].

\section{Experimental}

\subsection{Reagents}

The $314.5 \mathrm{MHz}$ one-port, two terminal SAW resonator (RO2113A) used in this study was obtained from the Radio Frequency Monolithic Company (RFM, USA). The AT-cut AT-cut piezoelectric crystal with $8 \mathrm{MHz}$ frequency used in this study was obtained from the Tai Tien Electric Co., Ltd., Taiwan. Escherichia coli (ATCC 43886) obtained from the Food Industry Research and Development Institute (FIRDI, Hsinchu, Taiwan) was inoculated into nutrient broth (Difco) and incubated at $30^{\circ} \mathrm{C}$ overnight. The home-made reaction cell was constructed from a Teflon cup (i.d. $=5 \mathrm{~mm}$, depth $=20 \mathrm{~mm}$ ) and a teflon cover which held two gold electrodes (o.d. $=1 \mathrm{~mm}, L=10 \mathrm{~mm}$ ). L-Glycine, L-cysteine, L-leucine, L-tyrosine, L-methionine, L-histidine, Laspartate, L-lysine and L-arginine were purchased from Sigma Chemical Co. (St. Louis, USA).

\subsection{Experimental set-up}

Fig. 1 shows the experimental set-up. The sensor was constructed with a SAW-stabilized oscillator [26]. A pair of gold electrodes was connected to the SAW resonator in series. The oscillation signal was fed to a frequency counter with a coaxial cable (Fig. 1A) (HP 53131A, Hewlett-Packard, USA).

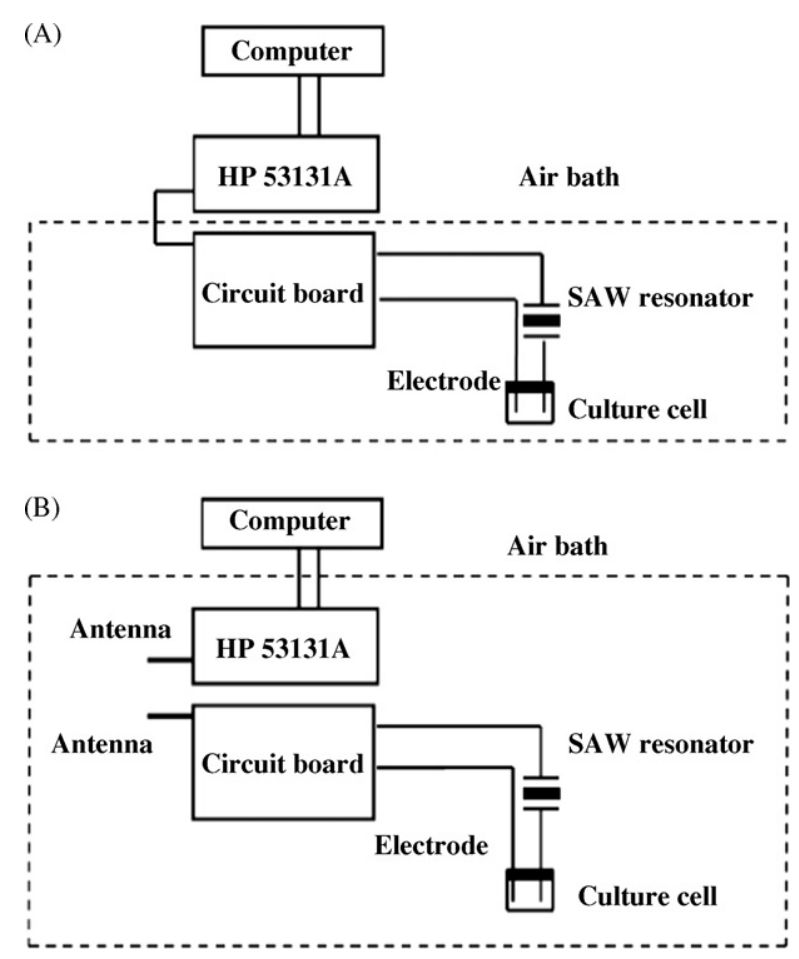

Fig. 1. Schematic diagram of the experimental set-up where (A) signal is fed to a frequency counter via a coaxial cable and (B) signal is transmitted wirelessly. 
The signal from the frequency counter was coupled to a PC (Pentium $600 \mathrm{MHz}$ ) using a PCMCIA-GPIB interface (GPIB National Instruments, Austin, TX, USA). Data acquisition and display were programmed using the LabVIEW 5.1 software package (National Instruments, Austin, TX, USA). Fig. 1B depicts the wireless experimental set-up. The design of the oscillator was the same as shown in Fig. 1A except that the signal from the oscillator was transmitted via the antenna to the frequency counter. The counter was separated from the sensor by approximately $20 \mathrm{~cm}$ and was used to receive the wireless signal. The series piezoelectric quartz crystal (PQC) sensor was constructed by connecting an AT-cut piezoelectric crystal with $8 \mathrm{MHz}$ frequency and a pair of gold electrodes in series. A homemade TTL oscillator was employed to drive the crystal.

\subsection{Procedure}

To eliminate cross-contamination during bacteria concentration monitoring experiments, the Teflon reaction cell and electrodes were washed and rinsed with distilled water and sterilized at $121{ }^{\circ} \mathrm{C}$ for $15 \mathrm{~min}$ before each incubation and prior to immersion in any of the solutions. Then, a volume of $0.1 \mathrm{~mL}$ milk sample mixed with $0.1 \mathrm{~mL}$ sterilized nutrient broth was added to the cell culture which was then fitted with the electrodes. Unless otherwise stated, the total volume was ca. $0.2 \mathrm{~mL}$ for each experiment. The conductive electrodes were immersed in the cell culture and connected to a $314.5 \mathrm{MHz}$ SAW resonator. The sensor was placed in an air bath at $30^{\circ} \mathrm{C}$ with precise temperature control within $\pm 0.2^{\circ} \mathrm{C}$. Unless otherwise stated, the measurements were taken half an hour after each inoculation of the cell cultures. The frequency was displayed and recorded by the computer until the incubation was completed. When a test is initially set up the user defines the detection criteria, normally $-10 \mathrm{~Hz}$ for most applications and when the rate of change of frequency exceed this pre-determined value the system will detect growth. The time required to reach the point of detection is referred to as detection time (DT) and is a function of the number of initial microbial population. Pour plates were prepared from these samples and incubated at $30^{\circ} \mathrm{C}$ for $24 \mathrm{~h}$. All colonies were counted $24 \mathrm{~h}$ after they were incubated. The detection times were plotted against the responses determined by the plate count method for the solutions in a calibration graph. For the determination of growth curve, a concentration of $E$. coli of ca. $10^{8}$ cells per milliliter (cells $/ \mathrm{mL}$ ) in the nutrient broth was used to prepare ca. $10^{1}$ to $10^{2}$ cells $/ \mathrm{mL} E$. coli in the aseptic nutrient broth. The concentration of $E$. coli in the dilution was confirmed by both plate counting method and SAW method. The growth curve of $E$. coli in this commercial aseptic nutrient broth at $30^{\circ} \mathrm{C}$ was obtained by determining the bacterial counts of sample every $2-3 \mathrm{~h}$.

\subsection{Principle}

The SAW resonator was used to control the frequency of a SAW resonator-stabilized oscillator. Fig. 2 shows the equivalent circuit model of a SAW resonator and its reactance characteristics with frequency. The circuit components $C_{\mathrm{m}}, L_{\mathrm{m}}, R_{\mathrm{m}}$ and

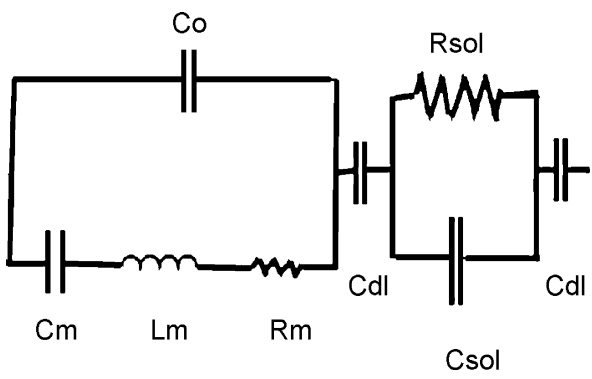

Fig. 2. The equivalent circuit of a SAW resonator. $C_{\mathrm{m}}, L_{\mathrm{m}}, R_{\mathrm{m}}$ and $C_{\mathrm{o}}$ are the motional capacitance, motional inductance, motional resistance and static capacitance, respectively, of the SAW resonator. $R_{\text {sol }}$ is the reciprocal of the solution conductance, $C_{\mathrm{sol}}$ is the permittivity of the solution, and $C_{\mathrm{dl}}$ is the double layer capacitance between the electrodes and the fluid establishing contact with the electrode.

$C_{\mathrm{o}}$ are the motional capacitance, motional inductance, motional resistance and static capacitance, respectively, of the SAW resonator. The components $C_{\mathrm{m}}$ and $L_{\mathrm{m}}$ determine the resonance frequency of the SAW resonator. When a load capacitor $\left(C_{\mathrm{L}}\right)$ is connected with the resonator in series, the frequency of the oscillator is changed by a frequency Df, where Df is $C_{\mathrm{m}} / 2\left(C_{0}+C_{\mathrm{L}}\right)$. The frequency is decreased with the increase of the $C_{\mathrm{L}}$.

Yoa et al. reviewed the characteristics of electrodeelectrolyte interfacial impedances when a pair of electrodes was connected with a SAW resonator in series [20]. For sinusoidal inputs, the terminal characteristics of an electrode have both a resistive $\left(R_{\mathrm{sol}}\right)$ and a reactive $\left(C_{\mathrm{sol}}\right.$ and $\left.C_{\mathrm{dl}}\right)$ component, where $R_{\mathrm{sol}}$ is the reciprocal of the solution conductance, $C_{\mathrm{sol}}$ is the permittivity of the solution, and $C_{\mathrm{dl}}$ is the double layer capacitance between the electrodes and the fluid establishing contact with the electrode $[8,28]$. When a pair of electrodes was connected to the SAW resonator in series and was placed within the bacterial culture solution of interest, microbial metabolism typically decreased both the resistance and the capacitance of the culture solution [5], causing a variation of the $C_{\mathrm{sol}}, C_{\mathrm{dl}}$ and $R_{\mathrm{sol}}$ of the circuit loop and thereby altering the frequency of the oscillator. These features permit the SAW resonator to be used for the real-time monitoring of bacterial growth within a sealed opaque container.

\section{Results and discussion}

\subsection{Frequency profile of the saw-stabilized oscillator}

The frequency profile of the SAW-stabilized oscillator is shown in Fig. 3. The oscillation signal was fed to a universal frequency counter by an axial cable (HP 53131A, USA). The noise level of the oscillator is $\pm 5 \mathrm{~Hz}$ (Fig. 3A). The relative frequency stability was $3.2 \times 10^{-8}$ based on the observed noise to oscillation frequency ratio over an average period of $20 \mathrm{~min}$. Value of frequency stability was the noise level divide by the frequency of the SAW device (314.5 MHz). Fig. 1B shows the wireless design of the oscillator, where the signal is transmitted by antenna to the frequency counter. The frequency profile obtained by the wireless transmission and the non-wireless transmission were almost identical to each other, except for the effects of EMI. A 

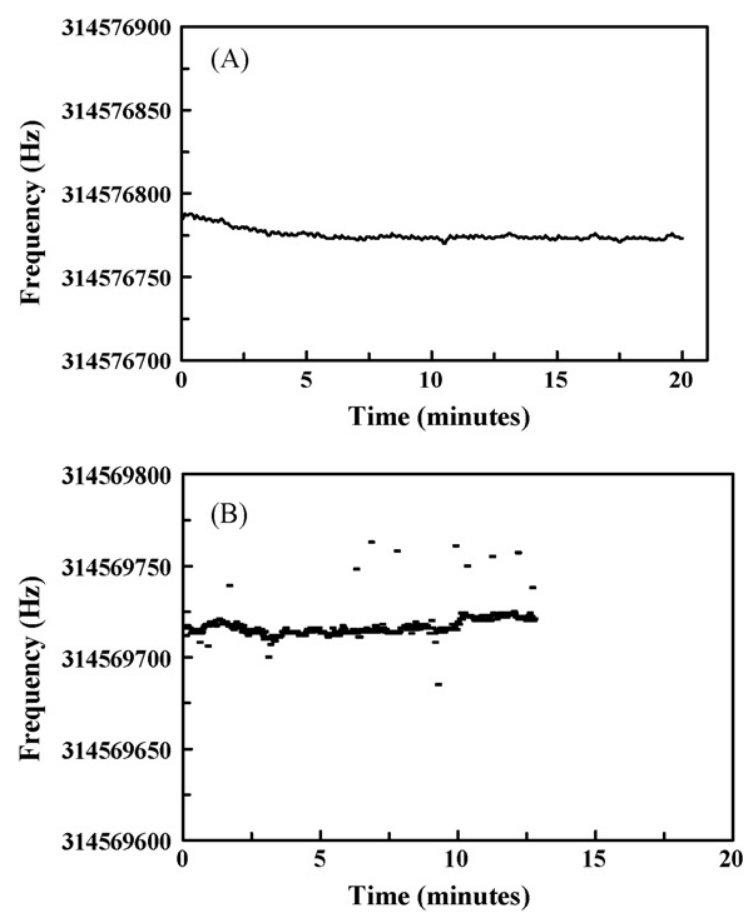

Fig. 3. Frequency profile of (A) the coaxial cable design and (B) the wireless design of the transmitter.

signal free from the effects of EMI is obtainable when the signal was fed into the counter via a coaxial cable. These findings indicate that the proposed SAW system has potential application for remote wireless monitoring of bacterial growth when the noise of EMI has been filtered.

The effect of temperature on the frequency of the oscillator was also studied. The frequency of the oscillator was steady within a temperature range from 25 to $40^{\circ} \mathrm{C}$ (Fig. 4), but became unstable when the temperature exceeded $40^{\circ} \mathrm{C}$. Based on the above results, a temperature of $30^{\circ} \mathrm{C}$ was selected for the following tests.

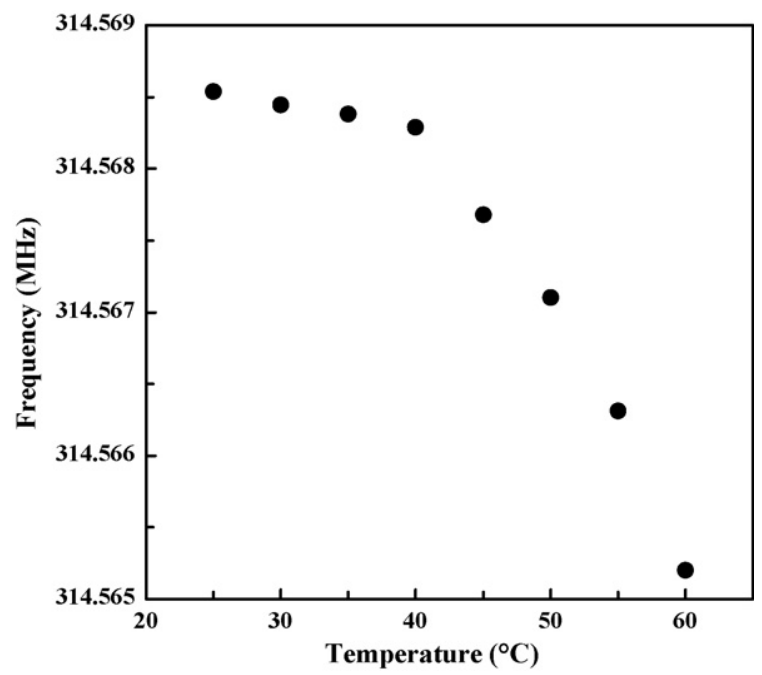

Fig. 4. Effect of temperature on the frequency of the SAW oscillator.

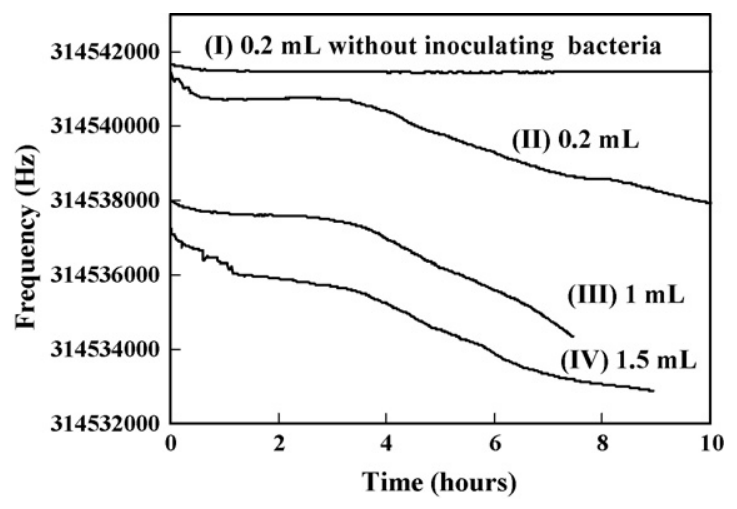

Fig. 5. Effect of sample volume on the response of the SAW sensor. The sample is a nutrient broth $(\mathrm{pH} 7.0)$ inoculated with ca. $10^{5}$ cells $/ \mathrm{mL}$ E. coli.

\subsection{Optimization of the bacterial growth monitoring system}

Fig. 5 (line I) shows the frequency profile of the SAW sensor when the electrodes were immersed in a reference culture cell containing sterile nutrient broth. The sensor response remained stable for more than $10 \mathrm{~h}$. The noise level of the sensor was $\pm 10 \mathrm{~Hz}$ and the relative frequency stability was $6.36 \times 10^{-8}$ based on the observed noise-to-oscillation frequency ratio over an average period of $10 \mathrm{~h}$. This result indicates that the physical and/or chemical factors unrelated to microbial growth of the media only affect the frequency of the sensor at a negligible level. On the contrary, the frequency decreases the frequency decreased slowly in the following $1 \mathrm{~h}$ when the electrodes were immersed in a cell culture containing bacteria inoculums. This may due to the bacteria and protein adsorbing on the electrode leading to an increase in the double layer capacitance $\left(C_{\mathrm{dl}}\right)$, resulting in the frequency decreased [6]. After equilibrium, the sensor response remained stable for a period of time hours (Fig. 5, line I). The stable time depend on the initial concentration of bacteria in the cell culture (Fig. 5, lines II and III).

A further study was carried out to investigate the effect of sample volume on the response of the sensor. The detection time of 0.2 and $1.0 \mathrm{~mL}$ sample volume were 2.7 and $3.0 \mathrm{~h}$, respectively (Fig. 5, lines II and III). Both lines have the same frequency profile model. A sample of $1.0 \mathrm{~mL}$ or larger than $1.0 \mathrm{~mL}$ required a longer time for the sensor to reach a plateau (Fig. 5, line IV), A sample volume less than $0.2 \mathrm{~mL}$, however, was insufficient to perform the operation. The sample volume was selected as $0.2 \mathrm{~mL}$ for the following experiments.

The effect of peptone and/or glucose enrichment on the growth of E. coli was also studied. Peptone enrichment was effective to have a shorter detection time of $E$. coli culture. On the contrary, the glucose enrichment showed no effect. As the result of our experiment, the breakdown of peptone by microbial metabolism was responsible for the frequency shift. Table 1 shows the effect of various amino acids on the frequency shift of the SAW sensor. The amino acids with acid or basic residues (e.g. L-aspartic acid, L-lysine, and L-arginine) could lead to a great change in the conductivity of the media, thereby the frequency shift notably, whereas the neutral amino acids (e.g. L-glycine, 
Table 1

The effect of the concentration of different amino acid on the frequency of the SAW sensor

\begin{tabular}{lllc}
\hline Amino acid & \multicolumn{2}{l}{ Frequency $(\mathrm{Hz})$} & $\begin{array}{c}\text { Frequency } \\
\text { change }(\mathrm{Hz})\end{array}$ \\
\cline { 2 - 3 } & $0 \mathrm{mM}^{\mathrm{b}}$ & $10 \mathrm{mM}^{\mathrm{b}}$ & \\
\hline L-Glycine & 314.564737 & 314.564532 & 205 \\
L-Cysteine & 314.564157 & 314.563890 & 267 \\
L-Leucine & 314.565694 & 314.565502 & 192 \\
L-Tyrosine & 314.565700 & 314.565501 & 199 \\
L-Methionine & 314.564930 & 314.564807 & 123 \\
L-Histidine & 314.565010 & 314.564750 & 260 \\
L-Aspartate & 314.565958 & 314.564010 & 1948 \\
L-Lysine & 314.564312 & 314.563072 & 1240 \\
L-Arginine & 314.564375 & 314.563849 & 526 \\
\hline
\end{tabular}

a The sample volume was $0.2 \mathrm{~mL}$ and the sensor was placed in an incubator at $30{ }^{\circ} \mathrm{C}$ with temperature precisely controlled at $\pm 0.2^{\circ} \mathrm{C}$. Each data point is the mean value of three measurements.

b Amino acid concentration.

L-cysteine, and L-leucine) caused relatively low frequency shift. Fig. 6 shows the effect of $\mathrm{pH}$ on the frequency response curves of $E$. coli in nutrient broth at $30^{\circ} \mathrm{C}$. The SAW sensor was used at an inoculums level of $10^{4}$ cells. Notably, detection time did not occur if the media was adjusted to $\mathrm{pH} 5.5$ by adding $\mathrm{HCl}$. This phenomenon can be explained by the following arguments. First, as described in a previous article [21], in high conductive media, the relative changes in conductance in the solution were smaller than in a low conductive media. The media with $\mathrm{pH} 7.0$ could be classified as a low electrolytic conductivity medium. Thus, the relative changes in conductance in the solution were significant, thereby producing a decrease in the frequency response after several hours' incubation. However, the electrolytic conductivity of the media with pH 5.5 could be classified as high. Therefore, it should not be expected that a frequency decrease will be found in response to the growth of bacteria at $\mathrm{pH} 5.5$ media. Second, the growth rate of E. coli in $\mathrm{pH} 5.5$ was slower than that at $\mathrm{pH} 7.0$ (data not shown). Interestingly, comparison of the growth rate of the bacteria in $\mathrm{pH} 8.5$ with that of $\mathrm{pH} 7.0$ revealed that both had identical grow curve (data not shown). However, the DT was delayed when the $\mathrm{pH}$ of culture media was increased from $\mathrm{pH} 7.0$ to 8.5 . It is possible that the major

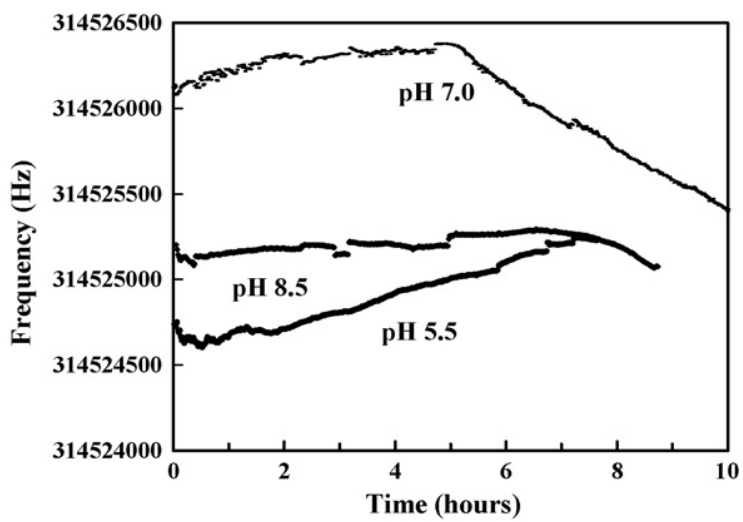

Fig. 6. Effect of $\mathrm{pH}$ on the response of the sensor at $30^{\circ} \mathrm{C}$. The sample is $0.2 \mathrm{~mL}$ nutrient broth inoculated with $10^{4}$ cells $/ \mathrm{mL} \mathrm{E}$. coli.

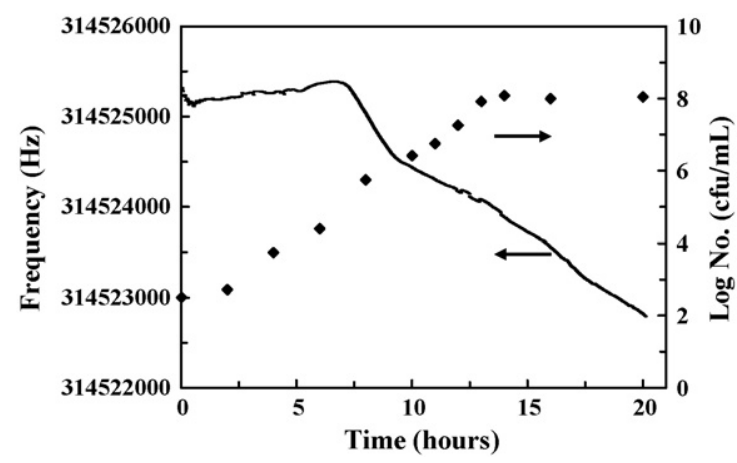

Fig. 7. Growth and frequency response curves of $E$. coli in nutrient broth $(\mathrm{pH}$ 7.0) at $30^{\circ} \mathrm{C}$ using the sensor with inoculums with $10^{2}$ cells $/ \mathrm{mL}$. The sample volume was $0.2 \mathrm{~mL}$.

protease produced by the $E$. coli in the present study is not alkaline protease. The protein breakdown by microbial metabolism was slow at high $\mathrm{pH}$. Thus, the changes in conductance in the solution were less significant. Therefore, $\mathrm{pH} 7.0$ of the culture medium was selected for the following tests.

\subsection{Detection time (DT) versus standard plate count}

The change in frequency of the oscillator when the electrodes were immersed in the culture was measured to monitor the growth of bacteria. Fig. 7 plots the growth and the frequency response against time after $E$. coli inoculation. A lag phase, a period of exponential growth, and a stationary phase were observed. The initial small change in frequency occurred in the seventh hour when the cell concentration reached $10^{5}$ to $10^{6}$ cells $/ \mathrm{mL}$. The microbial level associated with this change in frequency for such an impedance system is called the microbial threshold level. The microbial threshold level for our sensor was less than the conventional impedance method which shows a threshold level of $10^{6}$ to $10^{7}$ cells/mL $[12,13]$.

Fig. 8 shows the frequency response curves of the sensor with $10^{2}, 10^{4}$ and $10^{6}$ cells/mL inoculums size of $E$. coli in nutrient broth at $30^{\circ} \mathrm{C}$. The detection time was found exponentially

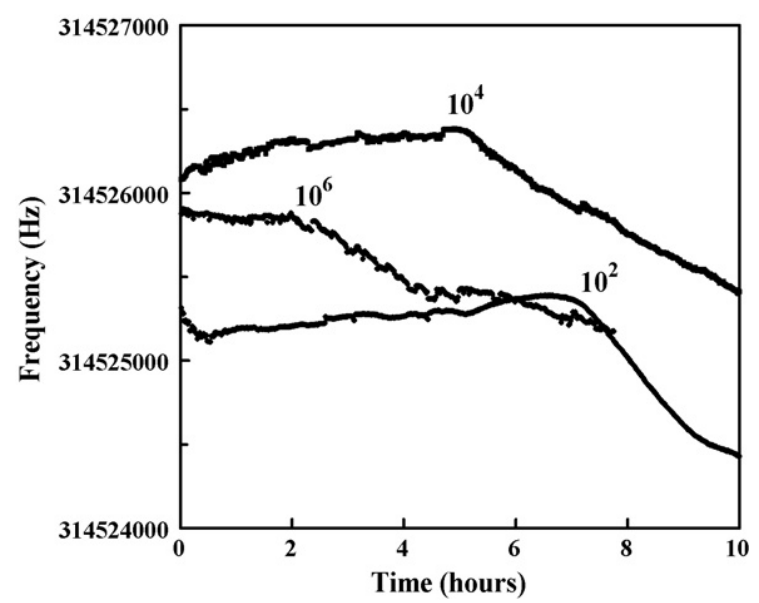

Fig. 8. The response curves of the sensor at $30^{\circ} \mathrm{C}$ in $0.2 \mathrm{~mL}$ nutrient broth $(\mathrm{pH}$ 7.0) inoculated with $10^{2}, 10^{4}$ and $10^{6}$ cells $/ \mathrm{mL}$ E. coli. 

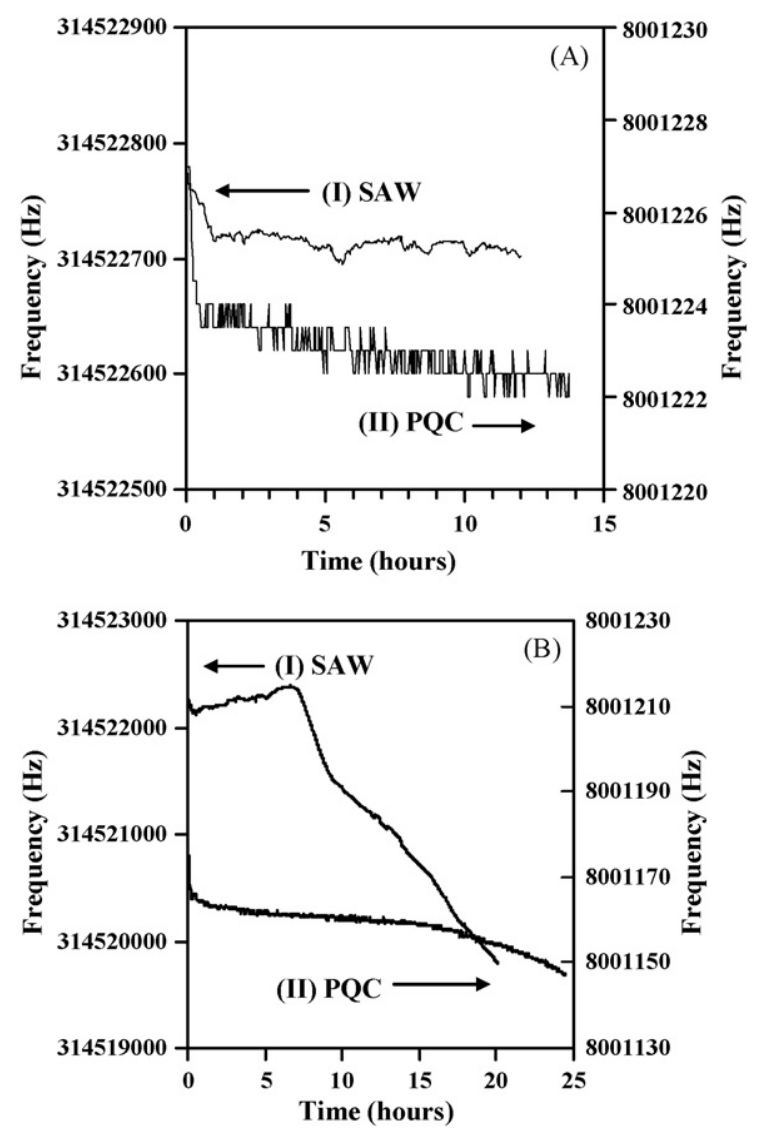

Fig. 9. (A) Noise trace of a SAW sensor (I) and a PQC sensor (II) in the sterile media. (B) Frequency response curves of SAW sensor (I) and SPQC sensor (II) of $E$. coli in nutrient broth with inoculums with $10^{2}$ cells $/ \mathrm{mL}$.

related to the inoculums size. The scattergrams of the detection time against the logarithm of the inoculums size of E. coli from $10^{2}$ to $10^{7}$ cells $/ \mathrm{mL}$ showed a linear curve. The regression equation was $Y=6.7692-0.6531 X(n=10)$ and the correlation coefficient $\left(R^{2}\right)$ was 0.924 . The relationship between the detection time and the concentration of $E$. coli became non-linear or undetectable when the inoculums concentration of E. coli was lower than $10^{2}$ cells $/ \mathrm{mL}$ (data not shown). Therefore, the detection limit of $10^{2}$ cells $/ \mathrm{mL}$ was determined. This finding is in agreement with previous reports using conventional impedance systems $[12,13,27,29]$.

Further studies involving pre-incubation of the sample before testing for lower bacterial concentrations may exploit whether these sensitivity limitations can be overcome [8].

\subsection{Comparison of SAW sensor with the PQC sensor for the determination of bacteria concentration}

The stability and sensitivity of proposed SAW sensor was compared with the PQC sensor (Fig. 9A). The SAW sensor and the PQC sensor have the same stability when the electrodes were immersed in a reference culture cell containing sterile nutrient broth. The noise level of the SAW sensor and PQC sensor were $\pm 10 \mathrm{~Hz}$ (relative stability of frequency $=6.36 \times 10^{-8}$ ) and $\pm 0.25 \mathrm{~Hz}$ (relative stability of frequency $=6.25 \times 10^{-8}$ ), respectively. The relative stability of frequency was based on the observed noise-to-oscillation frequency ratio over an average period of $10 \mathrm{~h}$. After the culture cell inoculated with E. coli, the SAW sensor shows a rapid initial rate change of the frequency signal $(400 \mathrm{~Hz} / \mathrm{h})$, with a large total frequency change and give a sharp inflexion at the DT, whereas the PQC sensor only shows a slow and shallow response $(0.25 \mathrm{~Hz} / \mathrm{h})($ Fig. 9B). This result reveals that the SAW sensor is superior to PQC sensor in determination of bacteria concentration. The noise level is the same for both SAW and PQC sensor. However, the resolution is higher for the SAW sensor due to its ultra high frequency. Furthermore, the SAW sensor was more sensitive than the PQC sensor based on the signal to noise ratio. When compared with conventional bacterial plating methods, this technique is relatively simple and, therefore, does not require the expertise of highly skilled technicians. Nevertheless, the detection limit of our sensor for $E$. coli was only $10^{2}$ cells $/ \mathrm{mL}$. Further investigation on the effect of pre-incubation of the sample may determine whether the sensor can be used in testing for lower bacterial concentrations. Another important feature of our SAW impedance system is the possibility of wireless transmissions of the sensor signal, which may enable the development of distinct application from conventional systems, such as in aseptic environments, which would be contaminated by a connecting wire or in hazardous environments. The wireless method may improve safety.

\section{Conclusions}

This study presents a novel design for a SAW impedance sensor. This device was used to detect the growth of E. coli. The impedance signal from the interface of the electrode in the bacterial culture solution was translated into a frequency signal by our SAW impedance sensor. An important advantage of our sensor is its lower microbial detection threshold number than traditional impedance methods. The microbial detection threshold in this study was $10^{5}$ to $10^{6}$ cells $/ \mathrm{mL}$. Hence, the sensor described in this paper is faster than instruments employing traditional impedance methods $\left(10^{6}\right.$ to $10^{7}$ cells $\left./ \mathrm{mL}\right)$. Although the proposed SAW sensor work at ultra high frequency, it possesses the same stability compare with that of lower frequency PQC sensor. Furthermore, the SAW sensor was more sensitive than the PQC sensor base on the signal to noise ratio. When compared with conventional bacterial plating methods, this technique is relatively simple and, therefore, does not require the expertise of highly skilled technicians. Nevertheless, the detection limit of our sensor for $E$. coli was only $10^{2}$ cells $/ \mathrm{mL}$. Further investigation on the effect of pre-incubation of the sample may determine whether the sensor can be used in testing for lower bacterial concentrations. Another important feature of our SAW impedance system is the possibility of wireless transmissions of the sensor signal, which may enable the development of distinct application from conventional systems, such as in aseptic environments, which would be contaminated by a connecting wire or in hazardous environments. The wireless method may improve safety. 


\section{Acknowledgement}

The authors would like to thank the National Science Council of the Republic of China for financially supporting this research under Contract No. NSC-92-2113-M-002-053-.

\section{References}

[1] J. Niza-Ribeiro, A.C. Louza, P. Santos, M. Lima, Monitoring the microbiological quality of raw milk through the use of an ATP bioluminescence method, Food Contr. 11 (2000) 209-216.

[2] M.R. Rosmini, M.L. Signorini, R. Schneider, J.C. Bonazza, Evaluation of two alternative techniques for counting mesophilic aerophilic aerobic bacteria in raw milk, Food Contr. 15 (2004) 39-44.

[3] I.K. Shitanda, Takada, Y. Sakai, T. Tatsuma, Amperometric biosensing systems based on motility and gravitaxis of flagellate algae for aquatic risk assessment, Anal. Chem. 77 (2005) 6715-6718.

[4] N.V. Kulagina, M.E. Lassman, F.S. Ligler, C.R. Taitt, Antimicrobial peptides for detection of bacteria in biosensor assays, Anal. Chem. 77 (2005) 6504-6508.

[5] C.J. Felice, R.E. Madrid, J.M. Olivera, V.I. Rotger, M.E. Valentinuzzi, Impedance microbiology: quantification of bacterial content in milk by means of capacitance growth curves, J. Microbiol. Methods 35 (1999) $37-42$.

[6] L. Yang, Y. Li, C.L. Griffis, M.G. Johnson, Interdigitated microelectrode (IME) impedance sensor for the detection of viable Salmonella typhimurium, Biosens. Bioelectron. 19 (2004) 1139-1147.

[7] J.W. Gardner, M. Cole, C.G. Dowson, P. Newton, G. Sehra, Smart acoustic sensor for the detection of bacteria in milk, in: Proceeding of the 3rd IASTED International Conference on Biomedical Engineering, vol. 458, 2005, p. 168.

[8] R. Firstenberg-Eden, J. Zindulis, Electrochemical changes in media due to microbial growth, J. Microbiol. Methods 2 (1984) 103-115.

[9] P. Silley, S. Forsythe, Impedance microbiology—a rapid change for microbiologists, J. Appl. Bacteriol. 80 (1996) 233-243.

[10] M. Wawerla, A. Stolle, B. Schalch, H. Eisgruber, Impedance microbiology: applications in food hygiene, J. Food Prot. 62 (1999) 1488-1496.

[11] A. Ur, D.F.J. Brown, Impedance monitoring of bacterial activity, J. Med. Microbiol. 8 (1975) 19-27.

[12] R. Firstenberg-Eden, Rapid estimation of the number of microorganisms in raw meat by impedance measurement, Food Technol. 37 (1983) 65-70

[13] D. Hardy, S.J. Kraeger, S.W. Dufour, P. Candy, Rapid detection of microbial contamination in frozen vegetables by automated impedance measurements, Appl. Environ. Microbiol. 34 (1977) 14-17.

[14] P. Candy, S.W. Dufour, S.J. Kraeger, Electrical impedance measurements: rapid method for detecting and monitoring micro-organisms, J. Clin. Microbiol. 7 (1978) 265-272.

[15] K.O. Kolquhoun, S. Timms, C.R. Fricker, Detection of Escherichia coli in potable water using direct impedance technology, J. Appl. Bacteriol. 79 (1995) 635-639.

[16] P. Neaves, M.J. Waddell, G.A. Prentice, A medium for the detection of Lancefield group D cocci in skimmed milk powder by measurement of conductivity changes, J. Appl. Bacteriol. 65 (1988) 437-448.

[17] K.G. Ong, J. Wang, R.S. Singh, L.G. Bachas, C.A. Grimes, Monitoring of bacteria growth using a wireless, remote query resonant-circuit sen- sor: application to environmental sensing, Biosens. Bioelectron. 16 (2001) 305-312.

[18] T. Deak, L.R. Beucha, Comparison of conductimetric and traditional plating techniques for detecting yeasts in fruit juices, J. Appl. Bacteriol. 75 (1993) 546-550.

[19] W. Welsch, C. Klein, M. von Schickfus, S. Hunklinger, Development of a surface acoustic wave immunosensor, Anal. Chem. 68 (1996) 2000-2004.

[20] S. Yoa, K. Chen, D. Liu, L. Nie, Circuit network analysis method applied to surface acoustic wave impedance system in liquids, Anal. Chim. Acta 294 (1994) 311-318.

[21] S. Yoa, K. Chen, L. Nie, Application of a surface acoustic wave sensor system for the determination of non-aqueous solutions and phase transitions in lipid multibilayers, Anal. Chim. Acta 289 (1994) 47-55.

[22] S. Yoa, K. Chen, F. Zhu, D. Shen, L. Nie, Surface acoustic wave sensor system for the determination of total salt content in serum, Anal. Chim. Acta 287 (1994) 65-73.

[23] D. Liu, K. Chen, K. Ge, L. Nie, S. Yoa, Study on the urease activity in different plant seeds and clinical analysis of urea in human body fluids by a surface acoustic wave enzyme sensor system, Fresenius J. Anal. Chem. 354 (1996) 114-117.

[24] K. Ge, D. Liu, K. Chen, L. Nie, S. Yoa, Assay of pancreatic lipase with surface acoustic wave sensor system, Anal. Biochem. 226 (1995) 207211.

[25] F.L. Dickert, P. Forth, W.-E. Bulst, G. Fischerauer, U. knauer, SAW devicessensitivity enhancement in going from $80 \mathrm{MHz}$ to $1 \mathrm{GHz}$, Sens. Actuators B 46 (1998) 120-125.

[26] RFM, Product data book. RF solutions for communications and computing. RF Monolithics, Inc., Texas, USA, 1997, A:17.

[27] S.B. Martins, M.J. Selby, Evaluation of a rapid method for the quantitative estimation of coliforms in meat by impedimetric procedure, Appl. Environ. Microbiol. 39 (1980) 518-524.

[28] W. Laureyn, D. Nelis, P. Van-Gerwen, K. Baert, L. Hermans, R. Magnee, J.J. Pireaux, G. Maes, Nanoscaled interdigitated titanium electrodes for impedimetric biosensing, Sens. Actuators B 68 (2000) 360-370.

[29] S. Gnan, L.O. Luedecke, Impedance measurements in raw milk as an alternative to the standard plate count, J. Food Prot. 45 (1982) 4-7.

\section{Biographies}

Ku-Shang Chang received his $\mathrm{PhD}$ degree in Microbiological and Biological Engineering from the Taiwan University, Taipei, Taiwan. He is currently an associate professor in the Department of Food Science at Yuanpei University of Science \& Technology, Hsinchu, Taiwan. His research interests include food science and chemical sensors.

Chen-Kai Chang received his PhD degree in Microbiological and Biological Engineering from the Taiwan University, Taipei, Taiwan. He is currently an assistant professor in the Department of Food Science at Chia Nan University of Pharmacy and Science, Tainan, Taiwan. His research interests include plant cell culture and chemical sensors.

Chien-Yuan Chen received his PhD degree in Life Science from National Taiwan University, Taipei, Taiwan and $\mathrm{PhD}$ of Engineering from The University of Tokyo, Japan. He is currently a professor in Microbiological and Biological Engineering from the Taiwan University, Taipei, Taiwan. His research interests include life science and chemical sensor. 